\title{
Effects of Chronic Use of Carbamazepine and Valproate on Cognitive Processes
}

\author{
A. M. L. Coenen, G. M. L. G. Konings, ${ }^{1}$ A. P. Aldenkamp, ${ }^{2}$ W. O. Renier, and \\ E. L. J. M. van Luijtelaar
}

\begin{abstract}
We investigated effects on cognitive processes of chronic use of carbamazepine (CBZ) and valproate (VPA) in a group of young patients with epilepsy. Scores on various neuropsychological tests were obtained from patients treated with two monotherapy regimens, one involving $C B Z$ and one involving VPA. In general, the cognitive profile of the two antiepileptic drugs (AEDs) was the same, except for some attention and memory aspects on which the VPA subjects scored better and for some motor tests which the CBZ group performed faster. The latter finding is in accord with results of studies reporting an increase in motor speed induced by CBZ. Furthermore, the observed impairments caused by both $\mathrm{CBZ}$ and VPA were relatively mild as compared with those caused by traditional AEDs such as phenytoin (PHT) and phenobarbital (PB). Key Words: Carbamazepine-ValproateCognition-Attention-Memory-Motor speed.
\end{abstract}

Patients with epilepsy often show cognitive impairments; memory problems and disturbances of attention and concentration are the most frequently reported disorders (1-4). The negative effects on cognition are attributed to factors such as structural brain changes, type of seizures, frequency of seizures, and ictal discharges. Recently,

Received April 1, 1994; accepted March 16, 1995.

From the Department of Psychology, University of Nijmegen, Nijmegen, ${ }^{1}$ Department of Pediatrics, University of Leiden, and ${ }^{2}$ Department of Child Neurology, University Hospital Nijmegen, Nijmegen, The Netherlands.

Address correspondence and reprint requests to Dr. A. M. L. Coenen at NICI, Department of Psychology, University of Nijmegen, P.O. Box 9104, 6500 HE Nijmegen, The Netherlands. however, the detrimental effects of antiepileptic drugs (AEDs) themselves have been emphasized (4-7); e.g., Andrewes et al. (8) reported that patients treated with phenytoin (PHT) monotherapy performed worse on memory tests than patients treated with carbamazepine (CBZ) monotherapy. In addition, Corbett et al. (9) described a relationship between the duration of AED therapy and cognitive decline for various drugs, noting only slight effects for valproate (VPA). Trimble and Thompson (10) observed negative effects on memory for PHT, phenobarbital (PB), and ethosuximide as compared with CBZ and VPA. Dam (11) attributed the negative effects of the former drugs to their more serious side effects.

In their review of the literature, the Committee on Drugs (12) concluded that CBZ and VPA should be considered first-choice medications. O'Dougherty et al. (13), however, reported that CBZ pro- 
duced impairments in short-term memory. Other evidence suggests that VPA also causes cognitive impairments (14-16). In accord with such findings Gallassi et al. $(17,18)$ showed that discontinuation of AEDs is associated with a diminution in cognitive impairments. Moreover, a reduction in dose or a shift from polytherapy to monotherapy also causes cognitive improvements $(7,19,20)$. Aldenkamp et al. (21) noted differential drug effects in a withdrawal study in children, showing that discontinuation of PHT had more positive effects on cognition than did discontinuation of CBZ or VPA. Nevertheless, Coenen et al. (22) reported improvement of scores on cognitive tests due to the discontinuation of CBZ as well as VPA. However, a direct comparison between the effects on cognition of CBZ and VPA has not yet been made. In this study, we investigated the cognitive profiles of these two first-choice medications in subjects treated either with CBZ or VPA as monotherapy.

\section{Methods}

In all, 19 patients with epilepsy, 5 young men and 14 young women, were recruited from three outpatient clinics located in three different regions of The Netherlands. The mean age of the patients was 16.2 years (range 12-22 years). The I.Q. of all subjects was $>80$, and all were from middle-class families. Epileptic seizures were not the consequence of cerebral lesions in any participant, as shown by negative computed tomography (CT) or magnetic resonance imaging (MRI) scans, and epileptic seizures were almost controlled, with a frequency of less than one seizure in 3 months. Matched on the basis of medication and for age and I.Q., subjects were divided into two groups: a CBZ (Tegretol) group $(\mathbf{n}=11)$ and a VPA (Depakine) group ( $n=8$ ). Most patients ( 7 in the $C B Z$ and 6 in the VPA group) had idiopathic generalized epilepsy, and a minority (4 in the CBZ group and 2 in the VPA group) had symptomatic localization-related epilepsy. Consequently, despite general differences in prescription of the two AEDs, we concluded that no differences existed between the groups in type and frequency of epilepsy. Moreover, there were no differences in the duration of existence of epilepsy between groups, which in all cases was $\sim 5-6$ years. The patients had also received AEDs for that time, and there were no changes in type of AED in that time. A matching criterion which could not be fully met was gender: All subjects in the VPA group were women. All relevant information about the patients is shown in Table 1.

All patients were tested in the morning for $2 \mathrm{~h}$ in a quiet room with minimal distraction. The test battery consisted of 10 tests measuring memory, attention, and concentration, as well as psychomotor performance and motor speed. The tests were the following: first, the Raven Progressive Matrices test to assess I.Q. (23); second, the Trailmaking test with trail $A$ and $B$, measuring psychomotor performance and mental and motor speed (24); third, the Complex Figure of Rey test for visuoper-

Table 1. Composition of the two groups

\begin{tabular}{lcc}
\hline \multicolumn{1}{c}{ Group } & VPA & CBZ \\
\hline No. of subjects & 8 & 11 \\
M & 0 & 7 \\
F & 8 & 4 \\
Education & 1 & \\
$\quad$ Primary school & 7 & 1 \\
$\quad$ Secondary school & 6 & \\
Type of epilepsy & 2 & 7 \\
$\quad$ Idiopathic generalized epilepsy & $15.7 \pm 2.7$ & $16.1 \pm 2.9$ \\
$\quad$ Localization-related epilepsy & $114.8 \pm 12.1$ & $110.6 \pm 11.6$ \\
Age (yr) & $4.7 \pm 2.9$ & $6.7 \pm 4.6$ \\
I.Q. & $550 \pm 350$ & $460 \pm 295$ \\
Duration of medication $(\mathrm{yr})$ & $65.2 \pm 20.8$ & $5.6 \pm 2.2$ \\
Daily drug dose $(\mathrm{mg})$ & & \\
Blood level of drug $(\mu \mathrm{g} / \mathrm{ml})$ & &
\end{tabular}

VPA, valproate; $C B Z$, carbamazepine.

Values are $\mathrm{n}$ or mean $\pm \mathrm{SD}$. There were no differences between the groups with regard to age, I.Q., and medication duration. 


\section{A. M. L. COENEN ET AL.}

ceptual organization and visual memory (25) (after patients copied this figure their delayed recall was tested after $15 \mathrm{~min}$ ); and fourth, the Finger Tapping test, a motor task in which both the right and left hand were tested. A self-constructed version was used, containing a light-weight button which had to be pressed as many times as possible in $10 \mathrm{~s}$. After a 15-min interval, patients were given the remaining six tests. In the fifth test, the Bourdon Dot Cancellation Test for sustained attention and regularity in performance (26), groups of 3,4, and 5 dots are presented, and configurations of 4 dots had to be cancelled as quickly as possible. The sixth test was a simple reaction time test in which subjects had to push a button as fast as possible after the onset of a tone. In the seventh test, the 15-Word test for auditory and verbal memory (25), lists of 15 words were presented five times, each with an immediate recall. The patients' delayed recall was tested after $20 \mathrm{~min}$. The eighth test was the Stroop Color-Word test for selective and focused attention $(25,27)$. The ninth was the fluency test for mental organization and retrieval of longterm memory (24), tested in a semantic modality in which examples of the category "trees" had to be named. The last test was the pegboard test for psychomotor performance (24).

After patients finished all tests, blood samples were taken to verify drug concentrations. Blood serum levels were within normal reference ranges: VPA subjects $65.8 \pm 20.2 \mu \mathrm{g} / \mathrm{ml}$ (normal range 45$105 \mu \mathrm{g} / \mathrm{ml}$ ); CBZ subjects $5.6 \pm 2.2 \mu \mathrm{g} / \mathrm{ml}$ (normal range $4-8 \mu \mathrm{g} / \mathrm{ml}$ ). No patient had either intoxication levels or levels that were below normal.

\section{Results}

Differences between scores for the VPA and the CBZ groups were analyzed by a two-tailed $t$ test for independent groups. All data are shown in Table 2. Statistically significant differences were noted in the attention tests. The Bourdon Dot Cancellation test showed that the VPA group worked more regularly (they had smaller SDs) and more accurately (they missed fewer correct configurations). In the Stroop Color-Word test, subjects in the CBZ group reacted faster on card 1 and made more errors on reading card 3 , the card with which interference is determined. With respect to the memory tests, the VPA group was significantly better in immediately recalling the total number of words after the word lists were presented three times. These differences decreased to marginal significant levels in the fourth and fifth trials and, in accord with this, there were no further significant differences between the two groups in delayed recall.

We also noted a difference between the two groups on motor performance. On the tapping test, the $C B Z$ group was superior in both-hands condition, significant for the right hand condition, and marginally significant for the left hand condition. In the simple reaction time test, the CBZ group also tended to perform better, with faster reaction times.

\section{Discussion}

In several tests involving attention, the VPA group performed better than the CBZ group. Subjects in the latter group had more omissions in the Bourdon Dot Cancellation test and more errors in the Stroop Color-Word test. Furthermore, results from the cancellation test indicate that the VPA group worked with more regularity: they showed significantly smaller SDs in this test. Fluctuations in attention and concentration in the $\mathrm{CBZ}$ group probably led to omissions in this task. CBZ may exert a more negative influence on attention and concentration than VPA. In accord with this possibility, Aman et al. (14) and Aldenkamp et al. $(21,28)$ demonstrated that VPA provides more effective protection against epileptiform activity. With VPA, then, it may be easier to maintain stable sustained attention because disruptive activity is absent, which might explain why VPA subjects also worked with more regularity. Furthermore, the $\mathrm{CBZ}$ group showed more inferior performance in tests that require memory function. Recall on the 15-word test in the early phase of learning was significantly poorer in the CBZ group, which is in accord with the finding in another field of research showing that the level of attention and the amount of memory consolidation are positively related (29).

In contrast to the superior performance evident in strictly cognitive effects of the VPA group, the $\mathrm{CBZ}$ group showed superior performance in tasks demanding pure motor speed. In the tapping task and in card 1 of the Stroop test, the CBZ group was superior in terms of speed measures. A similar tendency was evident in the simple reaction time task. 
Table 2. Means and $S D$ of the various test scores of the VPA and $C B Z$ groups

\begin{tabular}{|c|c|c|c|}
\hline Test & VPA & $\mathrm{CBZ}$ & $p$-Value \\
\hline \multicolumn{4}{|l|}{ Attention } \\
\hline \multicolumn{4}{|l|}{ Bourdon-Vos test } \\
\hline Mean line time (s) & $13.1 \pm 2.6$ & $12.2 \pm 2.2$ & 0.41 \\
\hline SD & $0.6 \pm 0.4$ & $1.4 \pm 1.1$ & $0.03^{a}$ \\
\hline Total time (min) & $7.2 \pm 1.4$ & $6.7 \pm 1.2$ & 0.40 \\
\hline Omissions & $1.6 \pm 1.5$ & $6.1 \pm 4.6$ & $0.01^{a}$ \\
\hline \multicolumn{4}{|l|}{ Stroop test } \\
\hline Time card 1 (s) & $48.6 \pm 5.0$ & $43.4 \pm 5.7$ & $0.05^{a}$ \\
\hline Time card $2(\mathrm{~s})$ & $62.4 \pm 11.8$ & $61.8 \pm 10.8$ & 0.91 \\
\hline Time card 3 (s) & $95.1 \pm 14.8$ & $88.2 \pm 28.1$ & 0.51 \\
\hline Errors card 3 & $0.1 \pm 0.4$ & $1.8 \pm 1.8$ & $0.01^{a}$ \\
\hline \multicolumn{4}{|l|}{ Trailmaking test } \\
\hline Trail A (s) & $32.4 \pm 8.1$ & $30.2 \pm 8.8$ & 0.59 \\
\hline Trail B (s) & $42.4 \pm 10.1$ & $42.5 \pm 14.5$ & 0.98 \\
\hline \multicolumn{4}{|c|}{ Memory } \\
\hline \multicolumn{4}{|c|}{ 15-Word test (No. of words) } \\
\hline Total recall 1-3 & $29.7 \pm 4.2$ & $25.5 \pm 4.5$ & $0.05^{a}$ \\
\hline Total recall $1-5$ & $55.1 \pm 5.9$ & $48.9 \pm 8.8$ & 0.08 \\
\hline Delayed recall & $12.3 \pm 2.5$ & $10.5 \pm 2.9$ & 0.19 \\
\hline \multicolumn{4}{|l|}{ Figure of Rey test } \\
\hline Score copy & $35.9 \pm 0.4$ & $35.7 \pm 0.6$ & 0.53 \\
\hline Score recall & $24.1 \pm 4.2$ & $21.5 \pm 7.0$ & 0.31 \\
\hline \multicolumn{4}{|l|}{ Fluency test } \\
\hline No. of items & $8.8 \pm 2.4$ & $6.1 \pm 2.8$ & 0.59 \\
\hline \multicolumn{4}{|l|}{ Motor speed } \\
\hline \multicolumn{4}{|l|}{ Finger tapping test $(\mathrm{n} / 10 \mathrm{~s})$} \\
\hline Left hand & $48.0 \pm 7.2$ & $55.8 \pm 10.5$ & 0.07 \\
\hline Right hand & $51.3 \pm 9.3$ & $67.1 \pm 15.0$ & $0.01^{a}$ \\
\hline \multicolumn{4}{|l|}{ Simple reaction time test } \\
\hline Mean time (ms) & $204.1 \pm 19.0$ & $182.1 \pm 40.9$ & 0.15 \\
\hline \multicolumn{4}{|l|}{ Pegboard test (s) } \\
\hline Mean time left hand & $9.0 \pm 0.5$ & $9.3 \pm 0.9$ & 0.42 \\
\hline Mean time right hand & $8.5 \pm 0.8$ & $8.3 \pm 0.8$ & 0.53 \\
\hline
\end{tabular}

VPA, valproate; $\mathrm{CBZ}$, carbamazepine.

${ }^{a} p<0.05$.

These data are consistent with several reports, all indicating that $C B Z$ increases motor speed $(13,30$ 33). Our present findings also indicate that $C B Z$ may have a positive influence on this parameter. Effects on speed, however, are not always observed. Smith et al. (20) reported a study in which PB, PHT, primidone, and CBZ were compared. Although better test scores for the latter drug were obtained, no significant speed differences were detected. A possible explanation for these contradictory findings might be a differential effect of CBZ on pure motor speed and speed in which more mental processes, such as attention and concentration, are involved. When mental speed or speed with fine motor performance or with eye-hand coordination is the more important factor in determining overall performance, as in the trailmaking and the pegboard test, the speed measures might be different. For card 1 of the Stroop test, an almost purely motor task that can be performed almost automatically, significant differences existed between the two groups. On the other hand, no group differences were evident for card 3 and, to a minor degree, for card 2, cards for which considerable attention and mental speed is required. In these cases, the facilitating effects of $C B Z$ are reduced by the negative effects on attention and the net effect is minor. This analysis indicates that a positive effect of CBZ may be restricted to motor speed rather than to cognitive function. Such a conclusion is only partially in accord with the claims for a psychotropic effect of CBZ (33-35).

If the present results are viewed in light of the complex and sometimes conflicting data in the literature, they suggest that both CBZ and VPA may have negative effects on cognition. These effects 
seem mild, however, in comparison to those of classic AEDs such as PHT or PB. The effects of both drugs, however, are found in different domains. Pure motor performance appears to profit in some way from CBZ as compared with VPA, but attention and memory are mildly affected by CBZ. On the other hand, although VPA does not have such effects on motor performance, it has less negative effects on attention and memory than CBZ.

Acknowledgment: Parts of this experiment were performed by Y. Tjong-A-Tjoe. Dr. P. Eling was helpful in composing the test battery.

\section{References}

1. Addy DP. Cognitive function in children with epilepsy. Dev Med Child Neurol 1987;29:394-7.

2. Loiseau P, Signoret JL, Strube E. Attention problems in adult epileptic patients. Acta Neurol Scand 1984;69:31-4.

3. Renier WO. Learning disabilities and behavioural problems in children with epilepsy. Wien Klin Wochenschr 1990;102: 218-22.

4. Thompson PJ, Trimble MR. Anticonvulsant drugs and cognitive functions. Epilepsia 1982;23:532-44.

5. Aldenkamp AP, Alpherts WCJ, Moerland MC, Ottevanger N, Van Parijs JAP. Controlled release of carbamazepine: cognitive side effects in patients with epilepsy. Epilepsia 1987;28:507-14.

6. Renier WO. Restrictive factors in the education of children with epilepsy from a medical point of view. In: Aldenkamp AP, Alpherts WCJ, Meinardi H, Stores G, eds. Education and epilepsy. Lisse: Swets and Zeitlinger, 1987:3-13.

7. Reynolds EH. Mental effects of antiepileptic medication: a review. Epilepsia 1983;24:85-95.

8. Andrewes DG, Bullen JG, Tomlinson L, Elwes RDC, Reynolds EH. A comparative study of the cognitive effects of phenytoin and carbamazepine in new referrals with epilepsy. Epilepsia 1986;27:128-34.

9. Corbett JA, Trimble MR, Nichol TC. Behavioral and cognitive impairments in children with epilepsy: the long-term effects of anticonvulsant therapy. J Am Acad Child Psychiatry 1985;24:17-23.

10. Trimble MR, Thompson PJ. Memory, anticonvulsant drugs and seizures. Acta Neurol Scand 1981;64:4-14.

11. Dam M. Summing up of the success so far gained through choice of drugs or combination of drugs. Acta Neurol Scand 1984;69:19-22.

12. Committee on Drugs. Behavioral and cognitive effects of anti-convulsive therapy. Pediatrics 1985;76:644-7.

13. O'Dougherty M, Wright FS, Cox S, Walson P. Carbamazepine plasma concentration: relationship to cognitive impairment. Arch Neurol 1987;44:863-7.

14. Aman MG, Wery JS, Paxton JW, Turbott SH. Effect of sodium valproate on psychomotor performance in children as a function of dose, fluctuations in concentration and diagnosis. Epilepsia 1987;28:115-24.

15. Butlin AT, Danta G, Cook ML. Anticonvulsant effects on the memory performance of epileptics. Clin Exp Neurol 1984;20:27-35.

16. Trimble MR, Thompson PJ. Sodium valproate and cognitive function. Epilepsia 1984;25:60-4.

17. Gallassi R, Lorusso S, Stracciari A, Morreale A, Procaccianti $G$, Baruzzi A. Withdrawal of phenobarbital and carbamazepine in epileptic patients: a preliminary neuropsychological report. Acta Neurol Scand 1986;74:59-62.

18. Gallassi R, Morreale A, Lorusso S, Procaccianti G, Lugaresi E, Baruzzi A. Comparison of cognitive effects in epileptic patients during monotherapy and withdrawal. Arch Neurol 1988;45:892-4.

19. Pedersen B, Dam M. Memory disturbances in epileptic patients. Acta Neurol Scand 1986;74:11-4.

20. Smith DB, Mattson RH, Cramer JA, et al., and the Veterans Administration Epilepsy Cooperative Study Group. Results of a nationwide Veterans Administration cooperative study comparing the efficacy and toxicity of carbamazepine, phenobarbital, phenytoin and primidone. Epilepsia 1987;28: $50-8$.

21. Aldenkamp AP, Alpherts WCJ, Blennow G, et al. Withdrawal of antiepileptic medication in children-effects on cognitive function: the multicenter Holmfrid study. Neurology 1993;43:41-50.

22. Coenen A, Keinecke A, Groothusen J, Van Luijtelaar G, Renier W. Absetzens antiepileptischer Therapie, insbesondere von Carbamazepin und Valproat, auf das Kognitive Verhalten von Kindern. In: Wolf P, ed. Epilepsie 89. Reinbek: Einhorn-Presse Verlag, 1990:367-74.

23. Raven JC. Guide to the standard progressive matrices: sets A, B, C, D, and E. London: Lewis, 1960.

24. Lezak MD. Neuropsychological assessment, 2nd ed. New York: Oxford University Press, 1983.

25. Rey A. Examen clinique en psychologie. Paris: Presses Universitaires de France, 1964.

26. Van Zomeren AH, Brouwer WH. Clinical neuropsychology of attention. New York: Oxford University Press, 1994.

27. Stroop JR. Studies on interference in serial verbal reactions. J Exp Psychol 1935;18:643-62.

28. Aldenkamp AP, Gutter Th, Beun AM. The effect of seizure activity and paroxysmal electroencephalographic discharges on cognition. Acta Neurol Scand 1992;86(suppl 140):111-21.

29. Ghoneim MM, Mewaldt SP. Benzodiazepines and human memory: a review. Anesthesiology 1990;72:926-8.

30. Aman MG, Wery JS, Paxton JW, Turbott SH, Stewart AW. Effect of carbamazepine on psychomotor performance in children as a function of drug concentration, seizure type, and time of medication. Epilepsia 1990;31:51-60.

31. Janke W, Ehrhardt KJ, Munch U. Behavioural effects of carbamazepine after single and repeated administration in emotionally labile subjects. Neuropsychobiology 1983;10: 217-27.

32. MacPhee GJA, Mitchell JR, Wiseman L, et al. Effect of sodium valproate on carbamazepine disposition and psychomotor profile in man. Br J Clin Pharmacol 1988;25:59-66.

33. Schmocker AM, Hermann H, Eisert H-G. Psychotropic action of Tegretol in healthy test subjects. In: Janz D, ed. Epileptology. Stuttgart: Georg Thieme, 1976:187-9.

34. Ciba-Geigy. Hat Tegretal eine psychotrope Wirkung? Basel; Ciba-Geigy, 1968.

35. Dalby MA. Antiepileptic and psychotropic effect of carbamazepine (Tegretol) in the treatment of psychomotor epilepsy. Epilepsia 1971;12:325-34. 